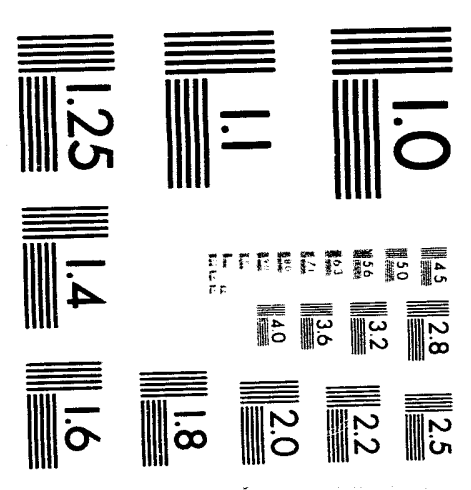



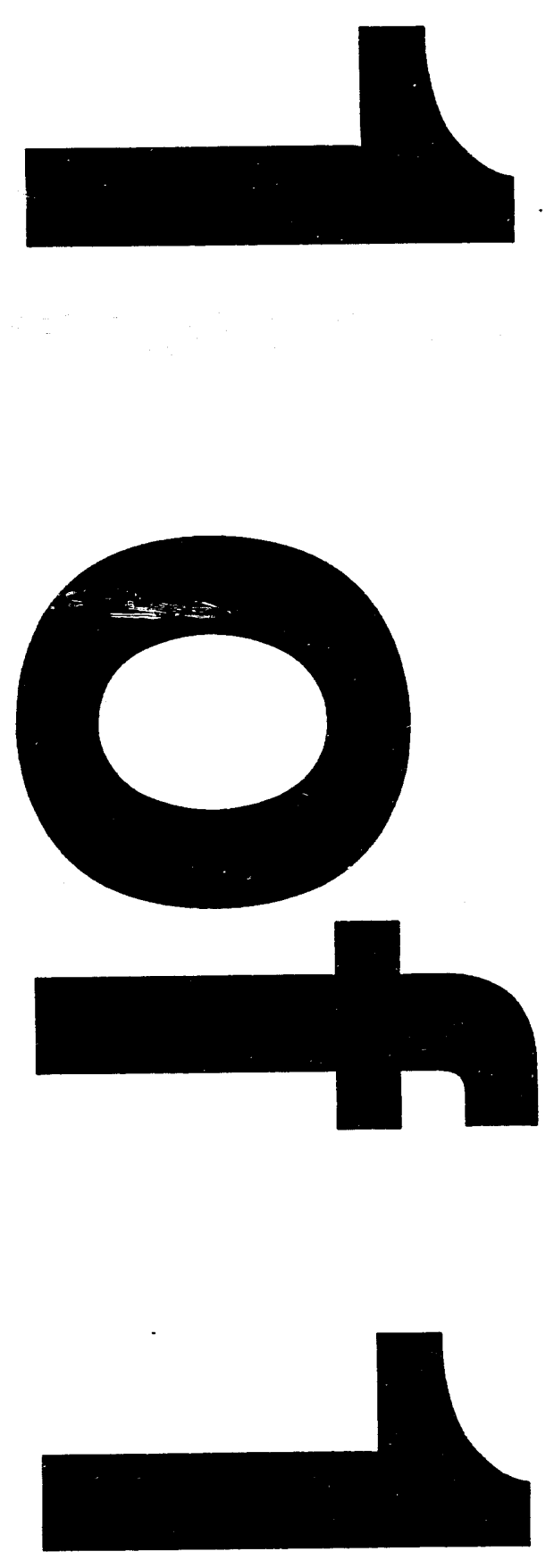


\title{
INVESTIGATION OF \\ COLLISIONAL EFFECTS WITHIN \\ THE BENDING MAGNET REGION \\ OF A DIII-D NEUTRAL BEAMLINE
}

by

D.N. KESSLER, R. HONG, and D.H. KELLMAN

This is a preprint of a paper to be presented at the 15th IEEE Symposium on Fusion Engineering, October 11-15, 1993, Cape Cod, Massachusetts, and to be printed in the Proceedings.

\author{
Work supported by \\ U.S. Department of Energy \\ Contract DE-AC03-89ER51114
}

GENERAL ATOMICS PROJECT 3466

OCTOBER 1993

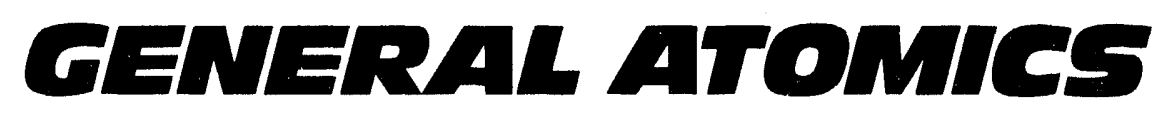




\title{
INVESTIGATION OF COLLISIONAL EFFECTS WITHIN THE BENDING MAGNET REGION OF A DIII-D NEUTRAL BEAMLINE
}

\author{
D.N. Kessler, R. Hong, and D.H. Kellman \\ General Atomics \\ P.O. Box 85608, San Diego, California 92186-9784
}

\begin{abstract}
The region between the pole faces of the DIII-D neutral beamline residual ion bending magnets is an area of transient high gas pressure which may cause beam defocusing and increased heating of beamline internal components due to collisional effects. An investigation of these effects helps in understanding residual ion trajectories and in providing information for studying the beamline capability for operation with increased pulse duration. Examination of collisional effects, and of the possible existence of space charge blow-up, was carried out by injecting deuterium gas into the region between the magnet pole faces with rates varying from 0 to 18 torr- $\ell /$ sec. Thermocouple and waterflow calorimetry data were taken to measure the beamline component heating and beam power deposition on
\end{abstract}

the magnet pole shields, magnet louvers, ion dump, beam collimators, and calorimeter. Data was also taken at gas flow rates varying from 0 to 25 torr- $\ell / \mathrm{sec}$ into the neutralizer cell and is compared with the magnet region gas injection data obtained. Results show that both collisional effects and space charge blow-up play a role in magnet region component heating and that neutralizer gas flow sufficiently reduces component heating without incurring unacceptable power losses through collisional effects.

\section{INTRODUCTION}

Each of the four DIII-D neutral beamlines contains two ion sources along with separate internal components for each beam path [1]. These components are the neutralizer cell, ion bending magnet, ion dump, beam dump, and beam collimators (Fig. 1). It is observed that certain

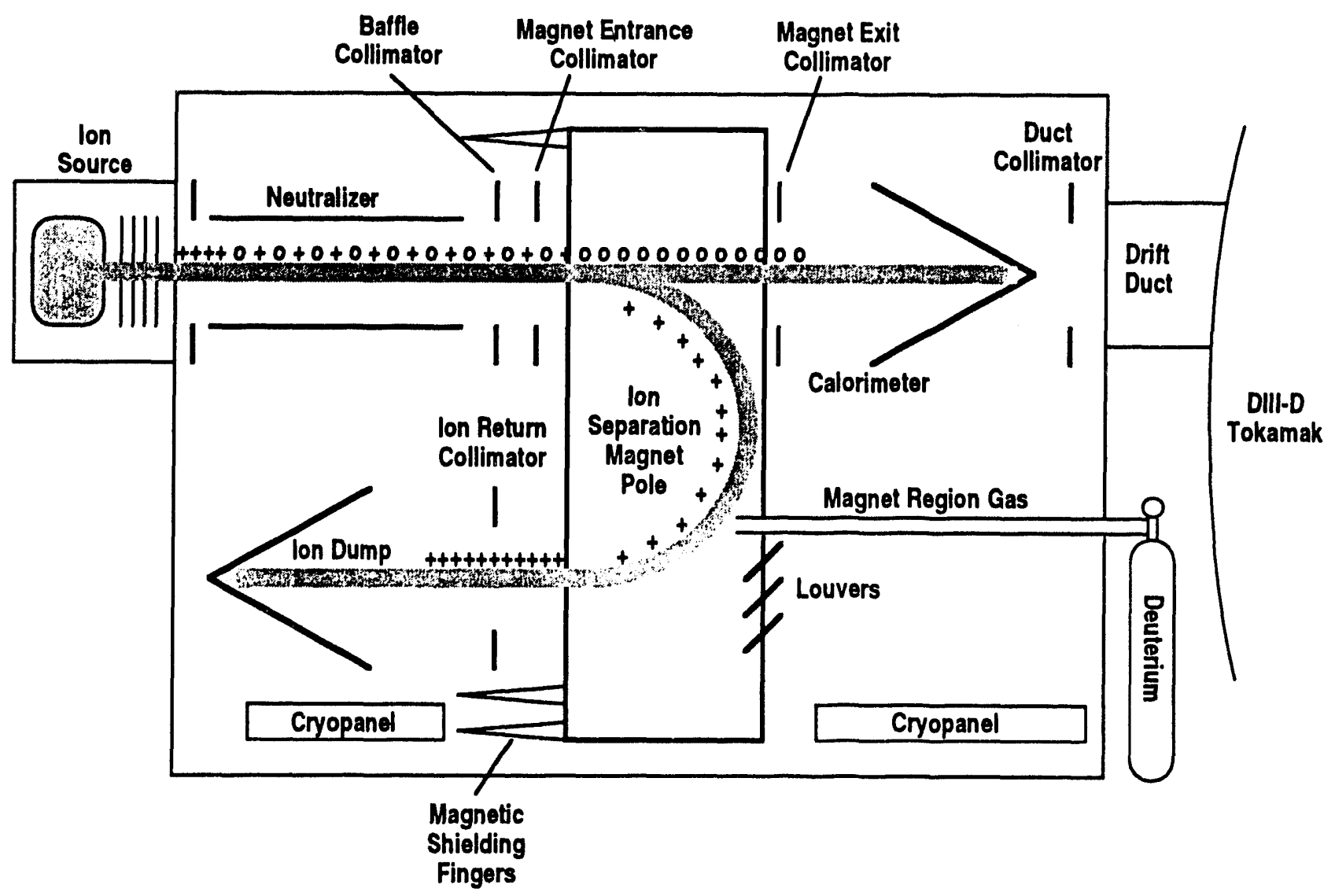

FIG. 1. Schematic representation of a DIII-D neutral beamline illustrating component locations and the magnet region gas feed modification.

Mamucript received October 12, 1893. This is a report of work sponsored by the U.S. Department of Energy under Contract No. DE-AC03-89ER51114. 
components have more beam power deposited on them than would be expected for a nonexpanding beam or a beam propagating in a vacuum. For instance, with no neutralizer gas flow it is observed that approximately $10 \%$ of the power extracted from an ion source is deposited on the bending magnet pole shields. In order to examine the effect of gas between the bending magnet pole faces, the capability to feed gas at various rates into that region was added to one beamline. With the existing ability to vary the neutralizer gas flow rate, two sets of data were taken with the following goals: (1) quantify the effects of neutralizer gas flow on component heating, (2) quantify the effects of varying amounts of gas between the magnet poles, and (3) look for the existence of space charge blow-up in the bending magnet region.

\section{EXPERIMENTAL SETUP}

\section{Neutralizer Gas System}

Deuterium gas is fed into the beamline neutralizer cell by an active feedback mass-flow controller which receives gas from the same reservoir as that used for ion source fueling. The flow rate and duration are programably controlled at the neutral beam control console where a digitized gas flow waveform may be displayed once a beam pulse is complete. During neutral beam operations the gas flow rate is normally set to 10 torr- $\ell / \mathrm{sec}$ and precedes beam extraction by several seconds to allow the gas flow to stabilize.

\section{Magnet Pole Region Gas System}

To accommodate this experiment a new gas line was installed in one of the beamlines allowing gas to be fed into the region between the bending magnet pole faces, Fig. 1. Flow through this line is regulated by a massflow controller identical to the one used to control gas flow into the neutralizer. As with the neutralizer gas system, flow rate and duration are programmed at the neutral beam control console.

\section{Thermocouple System}

Thermocouples are used extensively to monitor the temperature rise of beamline components. The thermocouple data acquisition system records two values for each thermocouple. The first value is a "baseline" reading just before a shot is fired. At a predetermined time after a shot has completed, typically $3.5 \mathrm{sec}$, a second reading is taken and compared to the first. The difference between these two readings is the temperature rise of the beamline component and is called $\Delta T$ in this paper.

\section{Waterflow Calorimetry System}

The Waterflow Calorimetry System [2] consists of differential thermocouple transducers which produce a voltage proportional to the difference in the monitored beamline component inlet and outlet cooling water temperatures. These voltages are read once a second by a
CAMAC digital voltmeter which is, in turn, read by a computer. Integrating the component temperature change waveform over time and multiplying by suitable constants yields the beam power deposited on the beamline component.

\section{Beam Parameters}

This experiment was performed with the following deuterium beam parameters: beam energy $=75 \mathrm{keV}$, beam perveance $=2.98 \mu \mathrm{p}$ (the units of perveance are ampere per volt ${ }^{1.5}$ ), beam pulse length $=1.00 \mathrm{sec}$, source gas flow rate $=20$ torr $-\ell / \mathrm{sec}$. The beam power extracted from the ion source (beam current times accelerator voltage) is called extracted beam power in this paper.

\section{NEUTRALIZER GAS FLOW SCAN}

\section{Effect on Neutralization Efficiency}

To achieve maximum neutral beam power and to reduce the heat loading on some beamline components, caused by excessive residual ions (ions which were not neutralized in the neutralizer), additional gas is fed into the neutralizer [3]. The measured neutralization efficiency at $75 \mathrm{keV}$ (deuterium beam) without neutralizer gas flow was $54.2 \%$, Fig. 2, and increased to $68.3 \%$ for a neutralizer gas flow rate of 25 torr- $\ell / \mathrm{sec}$.

At the normally used neutralizer gas flow rate of 10 torr- $\ell / \sec$ neutral beam power to the calorimeter increased by $13 \%$ as compared with no neutralizer gas flow. The rate of 10 torr $\ell / \mathrm{sec}$ is chosen because scattering causes the neutral beam power deposited on the calorimeter to be essentially constant at gas flow rates greater than about 15 torr- $\ell / \mathrm{sec}$, Fig. 2, while neutralizer heating, collimator heating, and induced gradient grid current, all continue to increase linearly with increasing neutralizer gas flow rate. At 10 torr- $\ell / \mathrm{sec}$ the neutral beam power deposited on the calorimeter is within $3 \%$ of the maximum value. With adequate gas flow in the neutralizer, the measured neutralization efficiency approaches the values calculated from a model by Kim and Haselton [4]. This is shown in Fig. 2. The theoretical

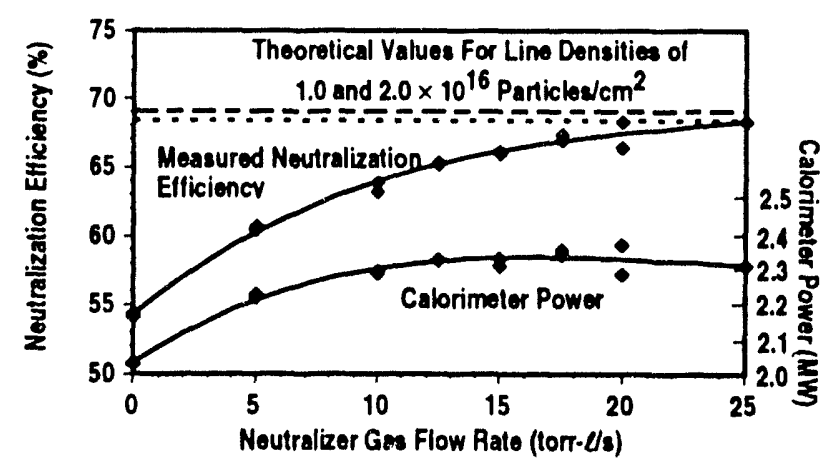

FIG. 2. Measured neutralization efficiency and calorimeter power as a function of neutralizer gas flow rate. 
values for the neutralization efficiency of a $75 \mathrm{keV}$ beam with a measured beam species mix of $77.5: 12.1: 10.3$ (\% full : $\%$ half : $\%$ third energy) into gas targets with line densities of $1.00 \times 10^{16}$ and $2.00 \times 10^{16}$ particle $/ \mathrm{cm}^{2}$ were obtained from this model.

\section{Changes In Component Heating}

When the ion bending magnet is energized, residual ions are deflected by 180 degrees into the ion dump. Due to the geometry of the ion removal system the residual ion beam is focused in one axis, at 90 degrees of deflection, causing a hot spot in the middle of the bending magnet pole shield. The bending magnet pole shield $\Delta T$ measurements are made using a thermocouple which reads at this location.

The plots of normalized power, Fig. 3, and normalized thermocouple $\Delta T$, Fig. 4 , show the neutral beam power to the calorimeter rising asymptotically to a limiting value (following the increase in neutralization efficiency) as the neutralizer gas flow rate is increased. Power to the bending magnet pole shield, magnet louvers, and ion return collimator, follows the decrease in residual ions (going as the inverse of the neutralization efficiency curve). The heating of the neutralizer cell and magnet entrance collimator increases linearly with increasing neutralizer gas flow rate irrespective of the asymptotic approach of the neutralization efficiency to

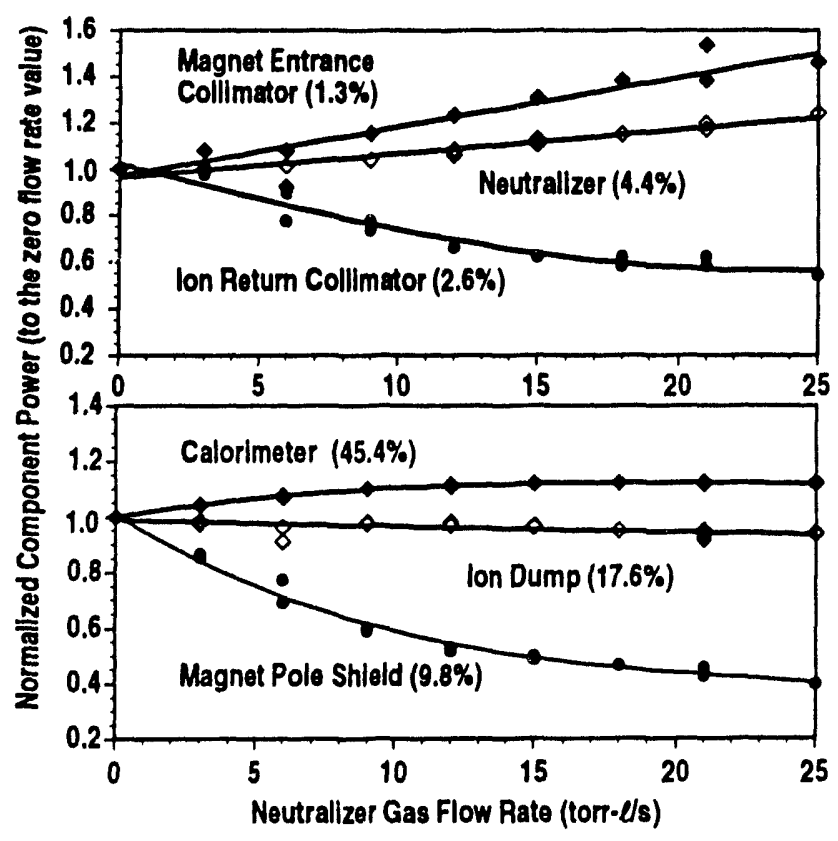

Fig. 3. Normalized power to six beamline components versus neutralizer gas flow rate. The plots are normalized to their values with no neutralizer gas flow, and the numbers in parentheses are the percent of extracted beam power deposited on the components with no neutralizer gas flow.

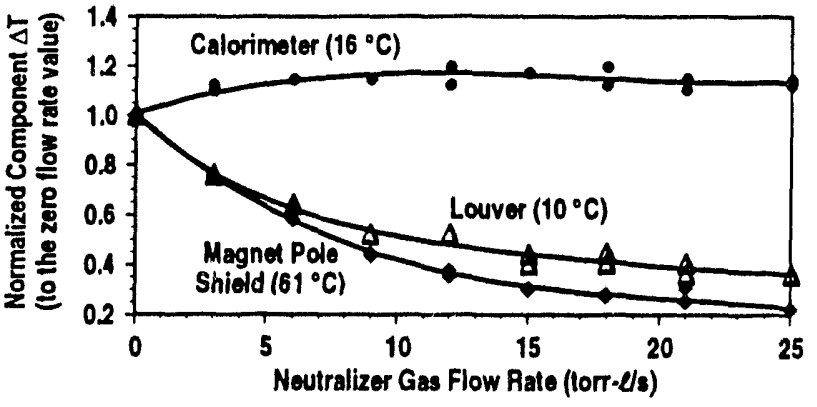

Fig. 4. Normalized temperature change for three beamline components versus neutralizer gas flow rate. The plots are normalized to their values with no neutralizer gas flow, and the numbers in parentheses are the component temperature increases with no neutralizer gas flow.

a constant value. This is attributed to increasing ionization in the neutralizer cell and the scattering of energetic beam particles.

An unusual result is that the fraction of beam power to the ion dump remained approximately constant instead of being inversely proportional to the neutralizer gas flow rate. Since the total beam power accountability is not changing (as measured by waterflow calorimetry), this indicates that residual ions are being lost to the magnet pole shield, louvers, and ion return collimator when little or no neutralizer gas is being used, possibly due to space charge blow-up in the bending magnet region. The density decrease of the residual ion beam with neutralizer gas flow and/or the presence of neutral gas in the bending magnet region may be mitigating the space charge blowup.

\section{MAGNET REGION GAS FLOW SCAN}

\section{Effect on Neutralization Efficiency}

In performing the magnet region gas flow scan, the neutralizer gas was valved out and gas was then fed into the region between the bending magnet pole faces with flow rates varying from 0 to 18 torr $-\ell / \mathrm{sec}$. Magnet region gas flow at the rates indicated had no effect on the fraction of neutral beam power deposited on the calorimeter, Fig. 5.

\section{Changes in Component Heating}

Plots of the normalized beam power for various beamline components, Fig. 5, and of the normalized thermocouple $\Delta T$ 's, Fig. 6, show that the power deposited on the magnet pole shield and louvers increases as the magnet region gas flow rate is increased from 0 to about 5 torr $-\ell /$ sec. The louver heating indicates that some residual ions are being neutralized in the bending magnet region and striking components in the bending plane. Residual ion beam scattering off of the gas to the magnet pole shield and louvers is also occurring. Above 5 torr- $\ell / \mathrm{sec}$ more power is being deposited on the ion 


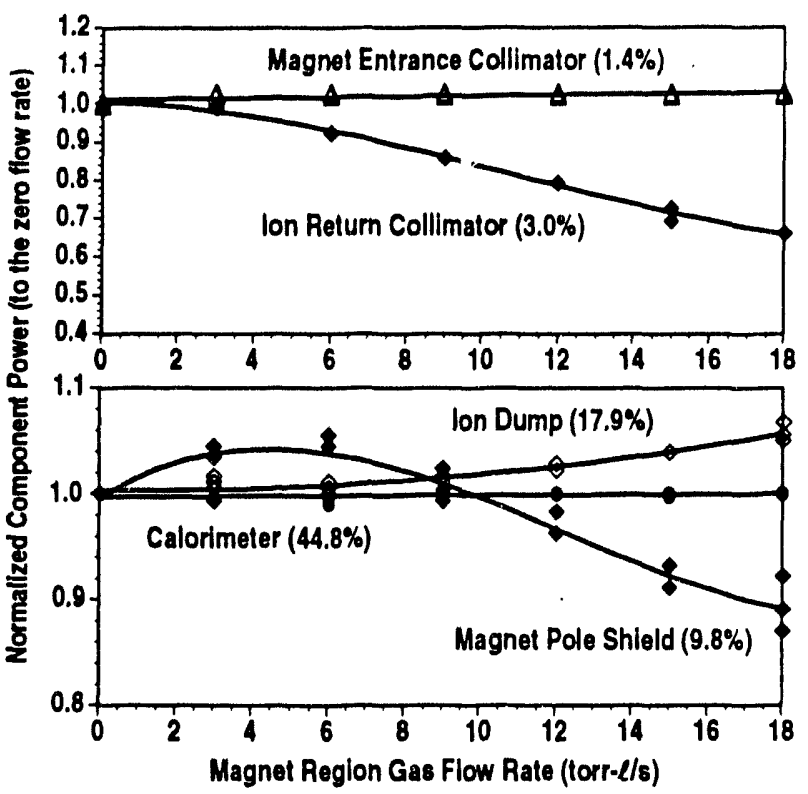

Fig. 5. Normalized power to five beamline components versus magnet region gas flow rate. The plots are normalized to their values with no magnet region gas flow, and the numbers in parentheses are the percent of extracted beam power deposited on the components with no magnet region gas flow.

dump, while power to the ion return collimator continues to decrease and power to the pole shield begins decreasing (even though scattering to the magnet region components is still taking place). Since the power deposited on calorimeter and magnet entrance collimator remained constant, this confirms that space charge blowup is present in the bending magnet region (with no neutralizer gas flow) and is being reduced (as indicated by the increase of number of ions deposited on the ion dump) when the magnet region gas flow rate exceeds 5 torr- $\ell /$ sec.

\section{CONCLUSIONS}

The use of neutralizer gas provides substantial benefits not only in increasing neutral beam power but in decreasing component heating in the bending magnet region. This is due to the decrease in the density and number of residual ions and to the presence of neutralizer gas in the bending magnet region which provides for the production of electrons. Both of these contribute to the mitigation of space charge blow-up.

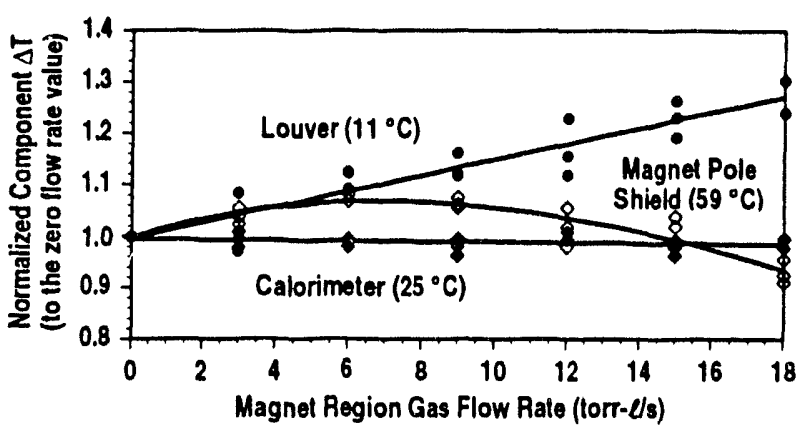

Fig. 6. Normalized temperature change for three beamline components versus magnet region gas flow rate. The plots are normalized to their values with no magnet region gas flow, and the numbers in parentheses are component temperature changes with no magnet region gas flow.

A neutralizer gas flow of 10 torr $-\ell / \mathrm{sec}$ increased the neutralization efficiency from $54.2 \%$ to $63.5 \%$ and increased the neutral beam power to the calorimeter by $13 \%$. This resulted in a $40 \%$ decrease in the residual ion beam power deposited on the magnet pole shield, and a $25 \%$ decrease in the residual ion beam power deposited on the ion return collimator. Injecting gas into the magnet region confirmed the presence of space charge blow-up by shifting residual ion beam power from the magnet pole shield and ion return collimator to the ion dump. It also increased the heating of some magnet region components due to residual ion beam scattering and neutralization.

\section{REFERENCES}

[1] A.P. Colleraine, D.W. Doll, R. Hong, J. Kim, A.R. Langhorn, C.H. Meyer, et al., "The DIII-D long pulse neutral beam system," in Proc. 11th IEEE Symp. on Fusion Engineering, pp. 1278-1281, 1885.

[2] J. Wight, R.M. Hong, J.C. Phillipa, R.L. Lee, A.P. Colleraine, and J. Kim, "Design and implementation of MecintoshCAMAC based system for neutral beam diegnostics," in Proc. 13th IEEE Symp. on Fusion Engineering, Oak Ridge, pp. 687690, 1889.

[3] J. Wight, R.-M. Hong, and J. Phillips, "Recent DII-D neutral beam calibration results," in Proc. 14th IEEE/NPSS Symp. on Fusion Engineering, San Diego, pp. 66-69, 1991.

[4] J. Kim and H.H. Haselton, "Analysis of particle species evolution in neutral-beam injection lines," J. Appl. Phys., vol. 50, p. $3802,1979$. 

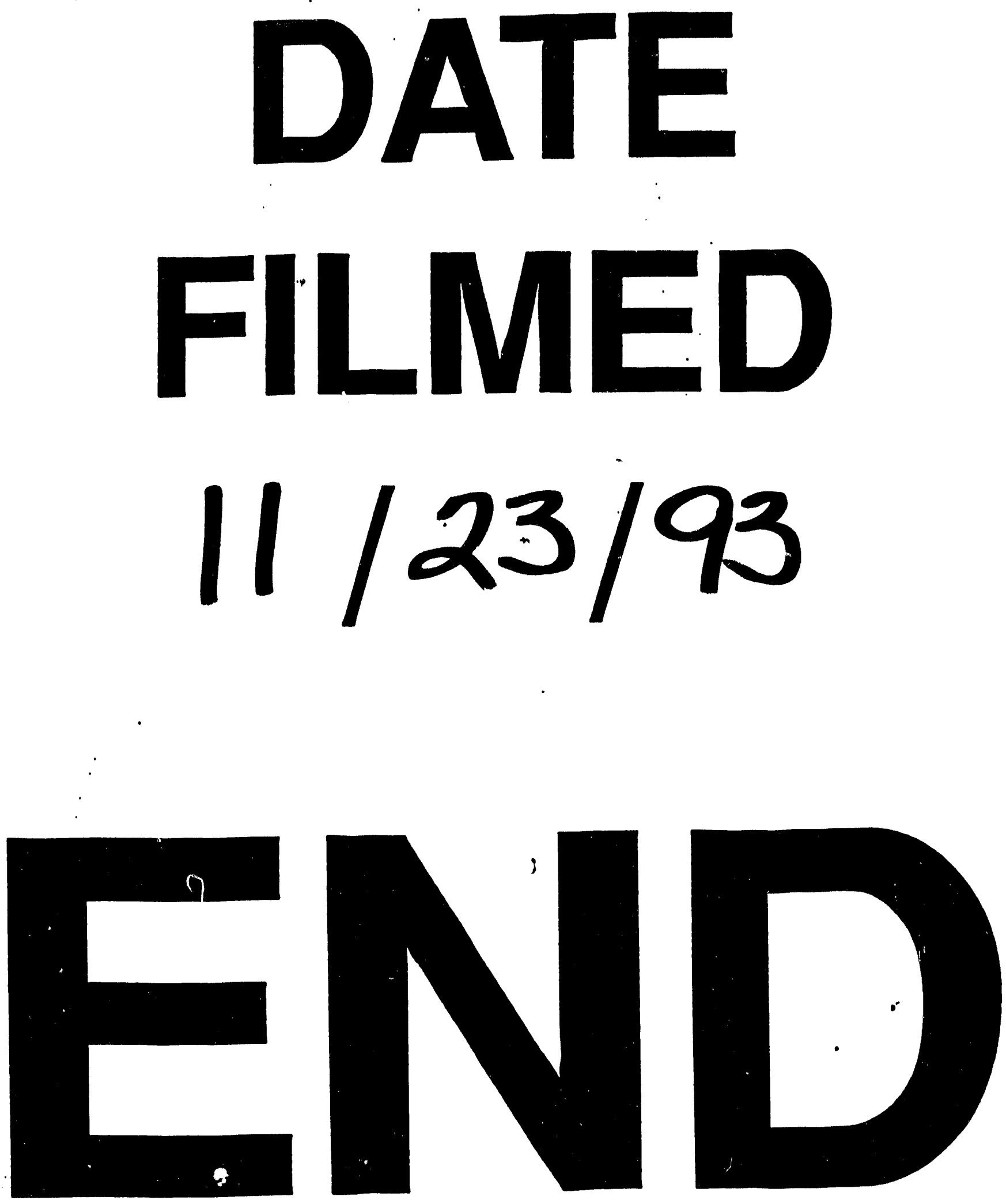
